ROCZNIKI PSYCHOLOGICZNE/ANNALS OF PSYCHOLOGY

2019, XXII, 2, 135-150

DOI: http://dx.doi.org/10.18290/rpsych. 2019.22.2-3

\author{
ŁUCJA BIELENINIK ${ }^{\mathrm{a}, \mathrm{b}}$ \\ CLAIRE M. GHETTI ${ }^{\mathrm{c}}$ \\ ${ }^{a}$ University of Gdańsk, Poland \\ Institute of Psychology \\ ${ }^{\mathrm{b}}$ GAMUT - The Grieg Academy Music Therapy Research Centre \\ NORCE Norwegian Research Centre AS, Bergen, Norway \\ ${ }^{\mathrm{c}}$ GAMUT - The Grieg Academy Music Therapy Research Centre \\ The Grieg Academy-Department of Music, University of Bergen \\ Norway
}

\title{
MUSIC THERAPY \\ FOR PRETERM INFANTS AND THEIR PARENTS: A PATH FORWARD FOR RESEARCH IN POLAND
}

\begin{abstract}
Music therapy has been internationally recognized as a health-promoting profession since the end of World War II, and music therapists have been conducting research in neonatal intensive care since the 1990s. Music therapy professional training was established in Poland in 1973 at the Music Academy in Wrocław, and Polish music therapists have recently begun to seek specialization to work within neonatal intensive care. The commencement of the multi-site international randomized controlled trial LongSTEP, Longitudinal Study of music Therapy's Effectiveness for Premature infants and their caregivers, has provided the impetus for Polish music therapists to begin offering music therapy services in neonatal intensive care. Thus, engagement in research marks the critical first step in the development of music therapy in neonatal care in Poland. This perspective article examines the current state of experimental research on music therapy in neonatal care and explores its implications for future research in Poland by (1) presenting the clinical
\end{abstract}

Corresponding author: ŁUCJA BIELENINIK-University of Gdańsk, Faculty of Social Sciences, Institute of Psychology, ul. Bażyńskiego 4, 80-309 Gdańsk, Poland; e-mail: lucja.bieleninik@ ug.edu.pl

Contributions: $Ł B$ and $\mathrm{CG}$ drafted the manuscript and approved the final version.

The authors received no specific funding for this work. ŁB was supported by a grant for young researchers and PhD students for 2018 from the University of Gdańsk.

CG was supported, in part, by a grant from the Research Council of Norway (RCN, project number 273534), under the program High-quality and Reliable Diagnostics, Treatment, and Rehabilitation (BEHANDLING). The funder had no role in the preparation of the manuscript. 
aspects of prematurity; (2) summarizing experimental research on music therapy in neonatal intensive care; (3) identifying gaps in the related evidence base; (4) discussing recent developments in international music therapy research; (5) contextualizing music therapy in the Polish neonatal health care system; (6) presenting advanced training in neonatal music therapy, and (7) discussing how culturally relevant aspects of neonatal settings in Poland might impact future research. There is preliminary evidence that music therapy plays a beneficial role for preterm infants and their primary caregivers during the neonatal period; however, research examining long-term impacts and longer-term intervention is needed. Researchers in Poland are poised to make a significant contribution to the international evidence base related to music therapy in neonatal care, and further exploration of particular facets of the Polish neonatal health care system that will impact the delivery of music therapy is warranted.

Keywords: prematurity; preterm neonates; parenting; neonatal care; music therapy; non-pharmacological interventions; psychosocial interventions.

\section{PREMATURITY}

Previous estimates, based on 184 countries including Poland, have highlighted a large global burden of prematurity worldwide. Each year, approximately 15 million neonates are born preterm (i.e., born before 37 weeks of gestation), corresponding to a global average prematurity rate of approximately $11.1 \%$ (Blencowe et al., 2012). Preterm birth rates in Poland oscillate around $6.7 \%$ (Chang et al., 2013), and in Europe they vary from 5 to $10 \%$ (Zeitlin et al., 2013). The rate of preterm birth in Europe is rising steadily (Blencowe et al., 2012), which makes prematurity a medical, psychological, social, and economic issue. Prematurity is the leading cause of death in the neonatal period and the second leading cause of death in children under 5 years of age (Liu et al., 2012). Prematurity is linked with associated illness (e.g., respiratory distress syndrome, retinopathy of prematurity, infection) and long-lasting sequelae (Blencowe et al., 2013). More than 1 million newborns die each year due to prematurity (Blencowe et al., 2013), and the consequences of prematurity extend beyond immediate treatment in the neonatal intensive care unit (NICU). Survivors are vulnerable to a variety of long-term consequences, including adverse physical outcomes (visual or hearing impairment, chronic lung disease of prematurity, long-term cardiovascular ill-health, and non-communicable diseases) and neurodevelopmental/behavioral disorders (mild disorders of executive functioning, moderate to severe global developmental delay, psychiatric/behavioral sequelae; Blencowe et al., 2013).

Prematurity also strongly impacts families. Due to unexpected delivery, premature birth is perceived as a stressful and traumatic event (Bieleninik, 
Bidzan, \& Salwach, 2009). Mothers of premature babies are at risk of postpartum depression and poorer well-being, which are the most important factors affecting early relationship between them and their infant in the postnatal period (Korja et al., 2008; Muller-Nix et al., 2004). Experiencing premature birth trauma might contribute to the adoption of a new parental role, that of being a "preterm baby's parent" - a role for which parents have not been prepared during pregnancy (Bieleninik, 2012).

After being delivered prematurely, babies start their lives in the NICU, where they are at risk of sensory overstimulation, which can negatively impact physiologic stability (Hassanein, El Raggal, \& Shalaby, 2013). At the same time, they are deprived of the natural sound environment that was present in utero, the lack of which can lead to compromised auditory brain development and delayed speech and language acquisition (McMahon, Wintermark, \& Lahav, 2012). In addition, preterm neonates with complex medical needs may experience physical separation from their parents. This separation might interrupt the development of healthy bonding and impact the subsequent formation of secure attachment (Bidzan, Bieleninik, Zdolska, \& Salwach, 2009). Parents who experience early physical separation from their preterm infants report higher levels of NICU-related stress (Franck, Cox, Allen, \& Winter, 2005). Being separated from their infant can negatively influence maternal sensitivity and responsiveness in subsequent interactions with their premature infants (Field, 2010). Family-centered approaches are therefore proposed that meet the infant's sensory needs while also promoting parent-infant bonding and decreasing parental stress.

\section{MUSIC THERAPY IN NEONATAL INTENSIVE CARE: AN OVERVIEW OF EXPERIMENTAL EVIDENCE}

The perinatal period is a crucial time for infant development, and the provision of developmentally appropriate and family-centered support within the NICU can improve long-term growth and development for high-risk preterm infants (AAP, 2004). Music therapy (MT) in neonatal care consists in "the informed use of music and aspects of a therapeutic relationship to promote optimal infant development and facilitate secure attachment with primary caregivers" (Bieleninik, Ghetti, \& Gold, 2016, p. 2). MT is provided by a qualified music therapist who has received additional training in therapeutic practice with infants and caregivers within the neonatal intensive care context. 
Evidence from systematic reviews and meta-analyses of controlled trials demonstrates that MT has positive effects on immediate and short-term outcomes for preterm infants and their parents (Bieleninik et al., 2016; Standley, 2012), though it is not yet consistently established as part of standard care. Systematic reviews and meta-analyses suggest that MT plays a beneficial role for preterm infants in relation to physiological parameters (e.g., regulating heart rate, improving oxygen saturation levels), feeding ability and weight gain (e.g., regulating sucking patterns, increasing breastfeeding volume and caloric intake), and regulating behavior states (Anderson \& Patel, 2018; Bieleninik et al., 2016; Hartling et al., 2009; Haslbeck, 2012; Hodges \& Wilson, 2010; Standley, 2012; van der Heijden et al., 2016). Mothers of preterm infants who engage in music therapy demonstrate decreased levels of parental stress, increased well-being, and improved coping; they also experience improved relation with their infants (Cevasco, 2008; Ettenberger et al., 2014; Ettenberger, Rojas Cárdenas, Parker, \& Odell-Miller, 2017; Loewy, Stewart, Dassler, Telsey, \& Home, 2013; Whipple, 2000). A meta-analysis comparing MT plus standard care with standard care alone demonstrated a large, statistically significant, overall effect size (Cohen's $d=0.82$ ), with positive impacts of MT on infant heart rate, behavior state regulation, oxygen saturation, sucking and feeding ability, and length of stay (Standley, 2012). Standley (2012) observed a negative correlation of effect size with birth weight and gestational age, suggesting that smaller and less mature preterm neonates (birth weight less than 1,000 grams and birth postmenstrual age less than 28 weeks) might benefit more from the impact of intervention. A recent rigorous meta-analysis involving 964 infant participants and 266 parent participants has confirmed a large favorable effect of MT on respiratory rate (mean difference, $-3.91 / \mathrm{min}, 95 \% \mathrm{CI}[-7.8,-0.03]$ ) and maternal anxiety (standardized mean difference, $-1.82,95 \%$ CI $[-2.42,-1.22]$; Bieleninik et al., 2016). The meta-analysis was restricted to randomized controlled trials (RCTs) with music therapist involvement, which resulted in a lower number of studies included than in previous meta-analyses (14 studies conducted in five countries: Australia, Brazil, Colombia, Israel, and the US), but ensured that the analyzed studies were rigorously designed. 


\section{GAPS IN THE EXPERIMENTAL EVIDENCE BASE FOR MUSIC THERAPY IN NEONATAL INTENSIVE CARE}

The current evidence base from controlled studies evaluating MT in the NICU is promising but indicates several areas that require additional research. The vast majority of RCTs (e.g., all but two of the 14 studies included in the meta-analysis; Bieleninik et al., 2016) focus on immediate or short-term outcomes for infants and caregivers. The lack of studies examining the long-term impact of MT with premature infants and their caregivers represents a major gap in the literature. One RCT of MT for mothers with or without their infants present demonstrated statistically significant increases in breastfeeding rates at 7-15 days post-discharge from hospital (Vianna, Barbosa, Carvalhaes, \& Cunha, 2011). A non-randomized controlled trial evaluated longer-term outcomes by assessing parent-infant interactions at one month post-treatment (Whipple, 2000) but found no significant differences between music therapy and control groups. Similarly, differences in breastfeeding rates in Vianna et al. (2011) at 30- and 60 -days post-discharge were not statistically significant. It is posited that MT can play an important role in promoting infant neurological development (Haslbeck, 2012; Standley \& Walworth, 2010), but without assessing infant neurological development over time it is difficult to make conclusive statements about such effects. Furthermore, it is difficult to know if the aforementioned beneficial short-term outcomes for preterm infants and their parents have lasting impacts for either infants or their parents. Policy makers, funders, and practitioners need to know if the benefits that music therapy demonstrates can lead to lasting gains and to what degree such gains can help offset costs to the individual, family, and the health care system that are associated with prematurity. Assessing long-term outcomes - those that occur years after the intervention - may be of keen interest to policy makers and funders who make decisions about whether or not to support the provision of music therapy as part of standard care.

There is also a lack of experimental studies evaluating the impact of longer-term MT intervention for preterm infants, as the majority of studies provide brief intervention periods from a single session to six sessions across the period of two weeks (Bieleninik et al., 2016). Within published RCTs, the longest MT intervention periods are approximately nine hour-long sessions aimed at mothers with or without their infants present (Vianna et al., 2011), and three to five 20-minute sessions for infants per week over the period of hospitalization (Cevasco, 2008). Studies exploring the extension of MT intervention past initial discharge from the NICU are only now being implemented (e.g., Ghetti et al., 
2019), and studies that have explored the benefits of longer-term MT for preterm infants once they have been discharged home are rare (e.g., Walworth, 2009). The differential impact of the frequency of MT intervention on infant and parental outcomes is reported in only one RCT, with a beneficial reduction in the length of gavage feeding reported for higher frequency of MT sessions (Standley et al., 2010). As the most extremely premature and medically complex infants can experience prolonged NICU hospitalization (defined as 120 days; Cotton et al., 2005), exploring the differential effectiveness of longer-term MT interventions - namely, from three weeks up to three months for eligible infants in the NICU - and of varying the frequency of MT intervention would help determine how therapeutic benefit correlates with the length and frequency of intervention. Factorial designs where participants are randomized to receive MT in NICU, after discharge, both, or neither help determine which phase of intervention is associated with the desired outcomes.

A few RCTs have examined the differential impact of various types of MT interventions, and those that compare live to recorded music report a more favorable effect for live music. Garunkstiene, Buinauskiene, Uloziene and Markuniene (2014), and Arnon et al. (2006) each explored the effects of different types of MT (live lullabies, recorded lullabies, and no-music sessions) over three consecutive days in prospective repeated measures crossover studies. The results suggested that live lullabies were more effective than recorded lullabies at facilitating deep sleep and decreasing heart rate (Arnon et al., 2006; Garunkstiene et al., 2014). Interventions that involve live music tailored specifically to the infant and parent enable music parameters to be matched to in-the-moment responses. Loewy et al. (2013) compared three types of interactive and individually-tailored MT ("song of kin" or parent-preferred lullaby, entrained heartbeat sounds via Gato Box instrument, and entrained breathing sounds via Remo ocean disc instrument) and found differential impacts of the three approaches on various short-term infant outcomes. One small RCT compared the outcomes of MT provided to infant and parent simultaneously during skin-to-skin care versus MT provided to the infant alone, but found no statistically significant differences (Ettenberger et al., 2014). As the aforementioned comparisons demonstrate different results for different MT interventions, the logical next step for MT research in neonatal care is the implementation of mechanistic studies that can elucidate the mechanisms underlying effective interventions.

Music therapists are increasingly attempting to include both parents actively in music therapy clinical practice (Mondanaro, Ettenberger, \& Park, 2016), and this is reflected in the research literature to a modest extent. Though parents are 
routinely included in MT clinical practice, it is only recently that parental perspectives and needs have been prioritized and systematically studied in MT research (Haslbeck, 2012; McLean, 2018). Fathers have not been exclusively targeted in any study so far, though they have been included among parental participants (Ettenberger et al., 2014; Loewy et al., 2013; McLean, 2018). As in the case of mothers, unexpected premature birth can interrupt the processes of fathers adopting their parental role too. Qualitative research illustrates that MT can play a crucial role in facilitating the formation of parental roles (McLean, 2018). Among many parental outcomes, only a decrease in maternal anxiety (Arnon et al., 2014; Ettenberger et al., 2014; Lai et al., 2006; Schlez et al., 2011) and parental stress (Loewy et al., 2013) as an effect of MT has been demonstrated in RCTs.

Despite the development of clinical practice that addresses the psychological needs of parents and families of preterm infants, there is still a lack of research examining the impact of MT in this area. Few RCTs have specifically assessed psychological outcomes for parents, and in those that do, reliable and valid psychological measures are seldom used (Bieleninik et al., 2016). A controlled clinical trial has demonstrated the beneficial impact of parent training in certain MT techniques to decrease infant and parent distress during their interactions in the NICU, though validated measures of parental psychological outcomes were not used (Whipple, 2000). MT has significantly decreased maternal anxiety in several studies, but evaluation of other psychological outcomes that might help determine whether MT can buffer against potential traumatizing effects of prematurity and related hospitalization is warranted (Bieleninik et al., 2016).

To date, very few studies have explicitly examined the role of MT in parent-infant bonding, and none has examined this outcome at longer term follow-up. The few studies that have engaged parents in MT in order to promote healthy bonding with their preterm infants report mixed results. Parent-infant bonding did not differ significantly for MT and control groups in two RCTs (Cevasco, 2008; Ettenberger et al., 2014), while parents who received MT reported an increase in the levels of bonding from pre- to post-test in a study using historic controls, but this difference was not statistically significant (Ettenberger et al., 2017). A systematic understanding of how MT contributes to the early relationship between neonates and parents is still lacking. 


\section{RECENT DEVELOPMENTS IN INTERNATIONAL MT RESEARCH DURING NICU STAY AND BEYOND}

Current experimental research is beginning to address major gaps in the knowledge base by assessing longer-term MT intervention and/or longer-term outcomes. Haslbeck, Bucher, Bassler, and Hagmann (2017) have registered a prospective single-center randomized controlled pilot trial (ClinicalTrials.gov, NCT02434224) of MT with standard care (SC) versus SC alone to assess impact on brain volume and long-term infant development. The pilot trial aims to assess the feasibility of the study protocol in advance of a multi-site definitive trial, and to explore whether MT improves brain growth and development at 38-42 weeks of corrected age, and neurobehavioral outcomes at 9 and 24 months and 5 years of age (Haslbeck et al., 2017). MT intervention consists in an interactive, needs-oriented, and infant-directed improvisational approach known as "Creative Music Therapy" (Haslbeck, 2014), which involves parents whenever possible. The study marks the first RCT of MT incorporating brain imaging and assessing the short- and long-term impact of MT on brain development.

An international multi-site $2 \times 2$ factorial pragmatic randomized controlled trial known as LongSTEP (Longitudinal Study of music Therapy's Effectiveness for Premature infants and their caregivers) is currently underway in five countries (Norway, Poland, Israel, Colombia, and Argentina; ClinicalTrials.gov, NCT03564184). Funded by the Research Council of Norway (RCN, project number 273534), LongSTEP aims to assess longer-term impact of MT on premature infants and their parents, as well as determine the effects of longer-term MT intervention (Ghetti et al., 2019). Like the Creative Music Therapy trial mentioned above, LongSTEP relies on an infant-directed, needs-oriented MT approach, but LongSTEP differs by placing primary emphasis on the music therapist supporting parents to be the prime provider of therapeutic singing. Parents' inherent musical and cultural resources are drawn upon as they are supported in the use of attuned, developmentally appropriate singing-based music interactions with their infant. The intervention is offered in two phases: MT three times a week during the length of NICU hospitalization, and/or MT approximately once a month during the first 6 months following discharge from NICU. The $2 \times 2$ factorial design examines the independent effect of each combination: MT during NICU hospitalization, MT for a 6-month period following discharge from NICU, both, or neither, along with interactions among them. The primary outcome is mother-infant bonding at 6 months of infant corrected age (CA), with secondary outcomes related to parental stress and anxiety, 
maternal depression, and infant general and socioemotional development at 6,12 and 24 months CA. The LongSTEP protocol was feasibility-tested in Norway and in Poland prior to initiating recruitment in those countries, and was found to be feasible and acceptable with minor adjustments (Ghetti et al., 2019).

\section{MT IN THE POLISH NEONATAL HEALTH CARE SYSTEM}

Health care in Poland is delivered through a publicly funded health care system called the Polish National Health Fund (Pol. Narodowy Fundusz Zdrowia). According to the Polish Constitution, all citizens are granted equal access to publicly funded health care services. Citizens are obligated to pay an insurance fee ( $9 \%$ deducted from personal income); however the government provides free health care to all young children, pregnant women, disabled people, and the elderly. At present, MT is not part of standard care in neonatal settings, and therefore MT is not financed through the national health fund. A cursory review of the websites of NICUs in Poland might suggest that several neonatal departments include music therapy in their offer of services. Upon further examination, however, it is clear that these hospitals are referring to music-based intervention consisting of music listening provided by medical personnel (e.g., nurses), not to music therapy offered by a trained music therapist and individually-tailored to the infant and the surrounding family. To our knowledge, music therapy for newborns, premature infants, and their family systems as offered by trained music therapists has not yet been introduced in Poland (L. Konieczna-Nowak, personal communication February 15, 2019).

Research related to the use of MT in neonatal health care services is only beginning to emerge in Poland; therefore, there is a call to undertake new research within this field, participate in international research, and host national research. A favorable circumstance for introducing MT into the neonatal intensive care context occurred in 2018, when Poland joined the above-mentioned international RCT-LongSTEP, first in the feasibility phase, and subsequently in the ongoing definitive trial. This opportunity was made possible by the cooperation between the Institute of Psychology of the University of Gdansk and the Department of Music Therapy of the Karol Szymanowski Academy of Music in Katowice. This domestic collaboration resulted from contact with several neonatal units and close cooperation with the Neonatology Department of the Municipal Hospital in Ruda Śląska, where the LongSTEP project is currently being conducted. Participation in this international research project provides an important first step to- 
wards initiating MT within Polish neonatal intensive care settings and introducing medical personnel to the potential of what MT might offer. The results of the study may inform how MT is implemented in NICUs in Poland.

\section{ADVANCED TRAINING IN NICU MT}

Before MT can be used in research within Polish NICUs or implemented within the Polish neonatal health care system, it is necessary to have music therapists who are specifically trained to work in NICUs. In Poland, there are currently five main higher education institutions (in Wroclaw, Łódź, Katowice, Krakow, and Lublin) that offer full-time or part-time bachelor and master degrees and additional postgraduate trainings in music therapy. The MT departments have a strong research team and collaboration contracts with international and national research communities. However, until now, there has been no organized neonatal intensive care MT training in Poland, and thus music therapists who sought such specialized training were forced to do so abroad.

Advanced training in the use of MT in neonatal intensive care is offered through two institutions in the United States (US) and one in Switzerland (Haslbeck \& Costes, 2011). The first training was developed by Jayne Standley at the National Institute for Infant and Child Medical Music Therapy at Florida State University in Tallahassee, Florida. The second one, called "First Sounds: Rhythm, Breath, \& Lullaby (RBL)," was established by Joanne Loewy at the Louis Armstrong Department of Music Therapy, Mount Sinai Beth Israel in New York. Friederike Haslbeck has developed a certification program for MT in neonatal care for music therapists from German-speaking countries. These training programs offer certification, and each involves collaborative links between a hospital and a university. Training consists of theoretical and practical components, often with follow-up supervision and/or an evaluative exam. The certification for German-speaking countries aims to create a training program that will promote transparency, professional competence, collaboration, and interdisciplinary exchange (Haslbeck \& Costes, 2011). As advanced training programs continue to develop, there is a growing awareness of the cultural variations that impact the practice of MT in various neonatal care settings. A forthcoming edited monograph will specifically address cultural variations across international MT practices in neonatal care (H. Shoemark, personal communication, March 18, 2019), a topic that is equally pertinent when considering the implementation of research studies. 


\section{CULTURALLY RELEVANT ASPECTS}

\section{OF NEONATAL SETTINGS IN POLAND}

When the condition of a premature neonate becomes stabilized, the period of "growth" begins. At the turn of the century, models of care for preterm infants in the NICU were updated. In many NICUs, the Newborn Individualized Developmental Care and Assessment Program (NIDCAP), Neurodevelopmental Treatment-Bobath (NDT) approach, or Vojta Therapy are part of standard care. In recent years, national healthcare guidelines (Borszewska-Kornacka, 2019) recommend that healthcare providers should support parents in engaging in the care of their neonates, whether born prematurely or not, and thus parental presence and involvement in the NICU is encouraged and supported. As a result of this policy, parents are encouraged to visit their children at any time and to participate in the feeding and care of the newborn. One of the most prevalent non-pharmacological interventions to involve parents is Kangaroo Care. This treatment is based on skin-to-skin contact between the child and the mother or father (or both). During the feasibility testing of the aforementioned LongSTEP project in Poland, medical staff observing the duration and frequency of parents' presence in the NICU noticed that mothers were highly involved in the caregiving and treatment process during NICU hospitalization, with lower engagement from fathers. The lack of fathers' presence in Polish NICUs, as well as the low level of their involvement in family-centered care, is most often a result of the fact that mothers take a maternal quota of maternity leave for the first 20 weeks directly after childbirth. Paternity leave (two weeks for the father; it may be used irrespective of the maternal leave-i.e., at the same time) can be taken until the child reaches 2 years of age, so not every father takes this benefit directly after delivery. In addition, the father is often seen as the head of the family and the person responsible for the family's financial situation, so he often continues full-time work after the birth of the child. Moreover, parental leave (32 weeks in the case of single birth and 34 weeks in the case of a multiple birth) is granted to both parents (parents may take leave at the same time or in turns), but mothers most often take a quota of parental leave beyond the NICU. In summary, we can say that one of the culturally relevant aspects regarding care in the postnatal period is that parental leave is taken to a greater extent by mothers. Thus, interventions in the postnatal period may easily reach mothers, but they may require special targeting to include fathers. Future MT research in Polish NICU settings should be consistent with the prevalent neurodevelopmental and family-centered models of care, which include encouraging both parents to take 
active and primary roles in caregiving during the NICU stay. Furthermore, as MT makes its initial way into Polish NICUs, it may be helpful to clearly frame MT as a means of supporting the innate musicality that unfolds during dialogue between the parent and the infant (Trevarthen, 2008), rather than as an externally applied intervention, in order for it to be accepted as a natural support to the parental role. When considering if both parents or just one will be invited to participate in a MT research study, typical patterns of parental leave can be considered to assure the feasibility of intervention implementation.

In a country like Poland, where MT is not yet part of standard care in NICUs or beyond, current research projects such as LongSTEP serve a critical function in establishing a culturally relevant evidence base that can inform the development of clinical services in the Polish neonatal healthcare system. Such studies can provide valuable information about what kind of MT approaches are relevant in this context as well as who should be prioritized to receive MT services. For example, if MT is found to be an effective means of promoting parent-infant bonding, then mothers who are identified as being at risk for experiencing problems with bonding might be prioritized for receiving MT.

Longitudinal interventional RCTs such as LongSTEP require interdisciplinary collaboration to assure quality of implementation, and thus can contribute to an increased understanding and awareness of the profession of MT in other health professionals within the specialized healthcare system. Such recognition and the establishment of interdisciplinary collaboration among music therapists and other allied health professionals may help assure smoother development of MT services in Polish NICU settings. Rigorously designed and implemented research studies create the evidence base that informs national healthcare guidelines. Such guidelines will then inform the implementation of services in the health care system. Improving research quality in MT is essential for enhancing ethical and scientific quality as well as promoting evidence-based practice development in Poland and beyond.

\section{CONCLUSION}

Previous experimental research suggests positive effects of music therapy for premature infants and their parents/caregivers, though methodologically rigorous studies with emphasis on long-term follow-up and child/parental psychological outcomes are needed. Studies examining the provision of MT beyond NICU hospitalization are also needed in order to determine the differential impact of 
the timing of MT services (Bieleninik et al., 2016). This perspective article focused on experimental research as well as implications and recommendations for future research are based on this framework. Additional gaps in the evidence base will be found when synthesizing the qualitative research in this area, and therefore the recommendations made in the present paper should not be considered exhaustive. Music therapists in Poland are engaging in a large-scale, international multi-site randomized controlled trial that will help to address critical gaps in the evidence base. To date, no randomized controlled study of MT has been conducted within the neonatal intensive care context in Poland (Bieleninik et al., 2016). Engaging in studies of high methodological quality will ensure that Polish music therapists and their interdisciplinary research collaborators help pave the way for the future development of evidence-based healthcare guidelines and clinical practice in Poland.

\section{REFERENCES}

AAP. American Academy of Pediatrics. (2004). Follow-up care of high-risk infants. Pediatrics, 114 (suppl 5), 1377-1397.

Anderson, D. E., \& Patel, A. D. (2018). Infants born preterm, stress, and neurodevelopment in the neonatal intensive care unit: Might music have an impact? Developmental Medicine \& Child Neurology, 60, 256-266. doi: 10.1111/dmcn.13663

Arnon, S., Diamant, C., Bauer, S., Regev, R., Sirota, G., \& Litmanovitz, I. (2014). Maternal singing during kangaroo care led to autonomic stability in preterm infants and reduced maternal anxiety. Acta Paediatrica, 103(10), 1039-1044. doi: 10.1111/apa.12744

Arnon, S., Shapsa, A., Forman, L., Regev, R., Bauer, S., Litmanovitz, I., \& Dolfin, T. (2006). Live music is beneficial to preterm infants in the neonatal intensive care unit environment. Birth, 33(2), 131-136. doi: 10.1111/j.0730-7659.2006.00090.x

Bidzan, M., Bieleninik, Ł., Zdolska, A., \& Salwach, D. (2009). Bond with a child in the prenatal period in case of prematurely born children. In K. Turowski (Ed.), Wellness and success (vol. 3, pp. 35-54). Lublin: NeuroCentrum.

Bieleninik, Ł. (2012). Dzieci przedwcześnie urodzone w percepcji matek [Preterm children as perceived by mothers]. Gdańsk: Harmonia Universalis.

Bieleninik, Ł., Bidzan, M., \& Salwach, D. (2009). The premature birth trauma and the parents' quality of life in the light of the S. Allen and A. Michalos's models. In G. Olchowik (Ed.), Wellness and success (vol. 2, pp. 39-52). Lublin: NeuroCentrum.

Bieleninik, Ł., Ghetti, C., \& Gold, C. (2016). Music therapy for preterm infants and their parents: A meta-analysis. Pediatrics, 138, e20160971. doi: 10.1542/peds. 2016-0971

Blencowe, H., Cousens, S., Chou, D., Oestergaard, M., Say, L., Moller, A. B., Kinney, M., \& Lawn, J., on behalf of the Born Too Soon Preterm Birth Action Group (2013). Born Too Soon: The global epidemiology of 15 million preterm births. Reproductive Health, 10(Suppl 1), S2. doi: 10-1186/1742-4755-10-S1-S2 
Blencowe, H., Cousens, S., Oestergaard, M. Z., Chou, D., Moller, A. B., Narwal, R., . . . Lawn, J. E. (2012). National, regional, and worldwide estimates of preterm birth rates in the year 2010 with time trends since 1990 for selected countries: A systematic analysis and implications. Lancet, 379, 2162-2172.

Borszewska-Kornacka, M. K. (2019). Standardy opieki medycznej nad noworodkiem w Polsce 2019. Zalecenia Polskiego Towarzystwa Neonatologicznego [Standards of neonatal medical care in Poland 2019. Recommendations of the Polish Neonatological Society]. Mielec, Poland: Oficyna Wydawnicza Press-Media.

Cevasco, A. M. (2008). The effects of mothers' singing on full-term and preterm infants and maternal emotional responses. Journal of Music Therapy, 45, 273-306.

Chang, H. H., Larson, J., Blencowe, H., Spong, C. Y., Howson, C. P., Cairns-Smith, S., . . Lawn, J. E., on behalf of the Born Too Soon preterm prevention analysis group (2013). Born Too Soon preterm prevention analysis group. Preventing preterm births: Analysis of trends and potential reductions with interventions in 39 countries with very high human development index. Lancet, 381, 223-234. doi: 10.1016/S0140-6736(12)61856-X

Cotton, C. M., Oh, W., McDonald, S., Carlo, W., Fanaroff, A. A., Duara, S., . . Goldberg, R. N. and NICHD Neonatal Research Network (2005). Prolonged hospital stay for extremely premature infants: Risk factors, center differences, and the impact of mortality on selecting a best-performing center. Journal of Perinatology, 25(10), 650-655.

Ettenberger, M., Odell-Miller, H., Cardenas, C. R., Serrano, S. T., Parker, M., \& Camargo Llanos, S. M. (2014). Music therapy with premature infants and their caregivers in ColombiaA mixed methods pilot study including a randomized trial. Voices: A World Forum for Music Therapy, 14(2). doi: 10.15845/voices.v14i2.756

Ettenberger, M., Rojas Cárdenas, C., Parker, M., \& Odell-Miller, H. (2017). Family-centred music therapy with preterm infants and their parents in the Neonatal Intensive Care Unit (NICU) in Colombia - A mixed-methods study. Nordic Journal of Music Therapy, 26(3), 207-234. doi: $10.1080 / 08098131.2016 .1205650$

Field, T. (2010). Postpartum depression effects on early interactions, parenting, and safety practices: A review. Infant Behavior and Development, 33, 1-6. doi: 10.1016/j.infbeh. 2009.10.005

Franck, L. S., Cox, S., Allen, A., \& Winter, I. (2005). Measuring neonatal intensive care unit-related parental stress. Journal of Advanced Nursing, 49(6), 608-615. doi: 10.1111/j.13652648.2004.03336.x

Garunkstiene, R., Buinauskiene, J., Uloziene, I., \& Markuniene, E. (2014). Controlled trial of live versus recorded lullabies in preterm infants. Nordic Journal of Music Therapy, 23(1), 71-88. doi: 10.1080/08098131.2013.809783

Ghetti, C. M., Bieleninik, Ł., Hysing, M., Kvestad, I., Assmus, J., Romeo, R., . . Gold, C. (2019). Longitudinal study of music therapy's effectiveness for premature infants and their caregivers (LongSTEP): Protocol for an international randomized trial. BMJ Open 2019;9:e025062. doi: 10.1136/bmjopen-2018-025062

Hartling, L., Shaik, M. S., Tjosvold, L., Leicht, R., Liang, Y., \& Kumar, M. (2009). Music for medical indications in the neonatal period: A systematic review of randomised controlled trials. Archives of Disease in Childhood. Fetal and Neonatal Edition, 94, F349-F354. doi: 10.1136/adc.2008.148411

Haslbeck, F. B. (2012). Music therapy for premature infants and their parents: An integrative review. Nordic Journal of Music Therapy, 21, 203-226. doi: 10.1080/08098131.2011.648653 
Haslbeck, F. B. (2014). Creative music therapy with premature infants: An analysis of video footage. Nordic Journal of Music Therapy, 23(1), 5-35. doi: 10.1080/08098131.2013.780091

Haslbeck, F. B., Bucher, H.-U., Bassler, D., \& Hagmann, C. (2017). Creative music therapy to promote brain structure, function, and neurobehavioral outcomes in preterm infants: A randomized controlled pilot trial protocol. Pilot and Feasibility Studies, 3(36). doi: 10.1186/s40814-017-0180-5

Haslbeck, F., \& Costes, T. (2011). Advanced training in music therapy with premature infants: Impressions from the United States and a starting point for Europe. British Journal of Music Therapy, 25(2), 21-33. doi: 10.1177/135945751102500203

Hassanein, S. M. A., El Raggal, N. M., \& Shalaby, A. A. (2013). Neonatal nursery noise: Practice-based learning and improvement. The Journal of Maternal-Fetal \& Neonatal Medicine, 26(4), 392-395. doi: 10.3109/14767058.2012.733759

Hodges, A. L., \& Wilson, L. L. (2010). Effects of music therapy on preterm infants in the neonatal intensive care unit. Alternative Therapies in Health and Medicine, 16, 72-73.

Korja, R., Savonlahti, E., Ahlqvist-Björkroth, S., Stolt, S., Haataja, L., Lapinleimu, H., Piha, J., Lehtonen, L., \& PIPARI study group (2008). Maternal depression is associated with motherinfant interaction in preterm infants. Acta Paediatrica, 97(6), 724-730. doi: 10.1111/j.16512227.2008.00733.x

Lai, H.-L., Chen, C.-J., Peng, T.-C., Chang, F.-M., Hsieh, M.-L., Huang, H.-Y., \& Chang, S.-C. (2006). Randomized controlled trial of music during kangaroo care on maternal state anxiety and preterm infants' responses. International Journal of Nursing Studies, 43, 139-146. doi: 10.1016/j.ijnurstu.2005.04.008

Liu, L., Johnson, H. L., Cousens, S., Perin, J., Scott, S., Lawn, J. E., . . Black, R. E., \& Child Health Epidemiology Reference Group of WHO and UNICEF (2012). Global, regional, and national causes of child mortality: An updated systematic analysis for 2010 with time trends since 2000. Lancet, 379(9832), 2151-2161. doi: 10.1016/S0140-6736(12)60560-1

Loewy, J., Stewart, K., Dassler, A. M., Telsey, A., \& Home, P. (2013). The effects of music therapy on vital signs, feeding, and sleep in premature infants. Pediatrics, 131(5), 902-918. doi: 10.1542/peds.2012-1367

McLean, E. (2018). An emergent exploration into the musical beginnings of parental identity across the neonatal journey (Doctoral dissertation, The University of Melbourne, Melbourne, Australia). Retrieved from http://hdl.handle.net/11343/214438

McMahon, E., Wintermark, P., \& Lahav, A. (2012). Auditory brain development in premature infants: The importance of early experience. Annals of the New York Academy of Sciences, 1252, 17-24. doi: 10.1111/j.1749-6632.2012.06445.x

Mondanaro, J. F., Ettenberger, M., \& Park, L. (2016). Mars rising: Music therapy and the increasing presence of fathers in the NICU. Music and Medicine, 8(3), 96-107.

Muller-Nix, C., Forcada-Guex, M., Pierrehumbert, B., Jaunin, L., Borghini, A., \& Ansermet, F. (2004). Prematurity, maternal stress and mother-child interactions. Early Human Development, 79(2), 145-158. doi: 10.1016/j.earlhumdev.2004.05.002

Schlez, A., Litmanovitz, I., Bauer, S., Dolfin, T., Regev, R., \& Arnon, S. (2011). Combining kangaroo care and live harp music therapy in the neonatal intensive care unit setting. The Israel Medical Association Journal, 13(6), 354-358.

Standley, J. (2012). Music therapy research in the NICU: An updates meta-analysis. Journal of Neonatal Network, 31, 311-316. doi: 10.1891/0730-0832.31.5.311 
Standley, J. M., \& Walworth, D. (2010). Music therapy with premature infants: Research and developmental interventions $\left(2^{\text {nd }}\right.$ ed.). Silver Spring, MD, US: American Music Therapy Association.

Standley, J. M., Cassidy, J., Grant, R., Cevasco, A., Szuch, C., Nguyen, J., . . Adams, K. (2010). The effect of music reinforcement for non-nutritive sucking on nipple feeding of premature infants. Pediatric Nursing, 36(3), 138-145.

Trevarthen, C. (2008). The musical art of infant conversation: Narrating in the time of sympathetic experience, without rational interpretation, before words. Musicae Scientiae, 15-46. https://doi.org/10.1177/1029864908012001021

van der Heijden, M. J. E., Oliai Araghi, S., Jeekel, J., Reiss, I. K. M., Hunink, M. G. M., \& van Dijk, M. (2016). Do hospitalized premature infants benefit from music interventions? A systematic review of randomized controlled trials. PLOS ONE, 11, e0161848. doi: 10.1371/journal.pone.0161848

Vianna, M. N., Barbosa, A. P., Carvalhaes, A. S., \& Cunha, A. J. (2011). Music therapy may increase breastfeeding rates among mothers of premature newborns: A randomized controlled trial. Jornal de Pediatria (Rio J), 87(3), 206-212. doi: 10.2223/JPED.2086

Walworth, D. D. (2009). Effects of developmental music groups for parents and premature or typical infants under two years on parental responsiveness and infant social development. Journal of Music Therapy, 46(1), 32-52.

Whipple, J. (2000). The effect of parent training in music and multimodal stimulation on parent-neonate interactions in the neonatal intensive care unit. Journal of Music Therapy, 37(4), $250-268$.

Zeitlin, J., Szamotulska, K., Drewniak, N., Mohangoo, A. D., Chalmers, J., Sakkeus, L., ... Blondel, B., \& the Euro-PEristat Preterm Study Group (2013). Preterm birth time trends in Europe: A study of 19 countries. BJOG, 120(11), 1356-1365. doi: 10.1111/1471-0528.12281 